\title{
An Improved Personal Security System
}

\author{
Sujata Punait*, A.R Askhedkar** \\ *(Department of Electronics \& Telecommunication, MIT COE Pune, Maharashtra \\ ** Department of Electronics \& Telecommunication, MIT COE Pune, Maharashtra
}

\begin{abstract}
In current period an enormous rise in crime against women can be observed and along with women even children, old age people are also suffering with inhuman activities as kidnapping. Women feel insecure roaming around the city alone, even the family members are always worried about their security till the time they get back safe. Many personal security solutions are available in the market in form of mobile applications and wearable devices as wrist bands, pendants, etc.. But all these already available solutions prove useless if the user is unable to trigger the device or mobile app in case of danger and also if device is lost or broken or battery goes low. This paper proposes a new improved personal security solution that will overcome these drawbacks of wearable devices and mobile applications making it more reliable solution for security.
\end{abstract}

Keywords: AI Thinker A7, Email Scheduler, Google docs, Raspberry Pi 3, Wearable device

\section{INTRODUCTION}

Today in the current worldwide situation, the prime question in each and every lady mind of every age group, considering the continually rising increment of issues on ladies badgering in later past, is just about her well being and security. The main thought frequenting in every girl mind is the point at which they will have the capacity to move uninhibitedly in the city indeed, even in odd hours without stressing over their security. Rape is the fourth most common crime against Women in India. According to latest National Crime Records Bureau (NCRB) 2013 annual report, 33,707 rape cases are reported across only India. The number of reported rape cases has been steadily increasing over the past decade. A step towards curbing incidents of this heinous crime is to develop a reliable security solution. Presently, women safety is not alone an issue but almost every individual is unsafe wether it is a child, man ,old age people.India is growing economically but still lot of attention is required in security field. Many times, especially in kidnapping or murder cases, the relatives of the victim and police comes to know about the incidence after several days or sometimes even after several weeks. Till that time investigation process for the police becomes difficult and chances of saving the person also reduces . This paper presents a review of various existing systems or devices that provides solution to personal security issues and a new improved personal security system is also presented overcoming the drawbacks of various already available systems or devices in the market. Most of the security solutions are built using wearable technology. The terms "wearable technology", "wearable devices", and "wearables" all refer to electronic technologies incorporated into items of clothing and accessories as bracelet ,pendant etc. which can comfortably be worn on the body. These wearable devices can perform many of the same computing tasks as mobile phones and laptop computers; however, in some cases, wearable technology can outperform these hand-held devices entirely. Generally, wearable technology will have some form of communications capability and that makes possible for the user access to information in real time. Data-input capabilities are also a feature of such devices, as is local storage.Some of the examples of wearable devices include watches, glasses, contact lenses, e-textiles and smart fabrics, headbands, beanies and caps, bracelets, and hearing aid-like devices that are designed to look like earrings. Security devices are also becoming wearable beacuse of ease in their usage in comparison to smartphones . A professor at Georgia Tech, Starner found a thumb rule for accesing devices called it the magic two-second rule. "If you can't get to a tool within two seconds," he says, "your use of it goes down exponentially." Even today, smartphones have trouble meeting that standard. By the time we extract them from our skinny jeans, swipe, type a passcode, and find our way to whichever app we wanted, the moment has usually passed. As Many a times existing solutions fails to prove useful in case of danger if our device is lost or broken or it fails due to some technical fault or person is unable to trigger the device or sometimes battery is discharged.This paper presents an improved personal security system which will overcome these drawbacks.

\section{EXISTING SOLUTIONS}

Many solutions are available for security of people. These solutions are in form of a device or available as an application which requires smartphones. 


\subsection{Existing solutions in form of a device}

This paper [1] suggests a new perspective to use technology to protect women. The system comprise of a normal cloves which when triggered, tracks the location of the victim using GPS (Global Positioning System) and sends emergency messages using GSM (Global System for Mobile communication), to three emergency contacts and the police control room. The system also consists of a screaming alarm that uses real time clock, to call out for help. This paper [2] presents device resembling a wrist band incorporated with pressure switch as an input which when activates shows the result .Screaming alarm and tear gas mechanism are included for self-defensing purpose and send location and messages to the emergency contacts and also figure out the attacker using live streaming video. Tear gas mechanism and live streaming video using webcam is combined together in the spectacles that act as a weapon of the smart technology. This paper [3] proposes a device which is the formed by combining multiple devices, hardware comprises of a wearable "Smart band" which continuously communicates with Smart phone that has access to the internet. The application is programmed and also it is loaded with all the required data which is Human behavior and reactions to varying situations like anger, fear and anxiety. This generates a signal which is transmitted to the smart phone. The software or application has access to GPS and Messaging services which is pre-programmed in such a way that whenever it receives emergency signal, it can send help request along with the location co-ordinates to the nearest Police station, relatives and the people in the near radius who have application. This action enables help instantaneously from the Police as well as Public in the near radius who can reach the victim with great accuracy. This paper[4] intends to present a practical and feasible combination of technologies for Woman Safety in the society . A social group, Guardians created by the system, where in to enlist verified users who can be contacted in case of emergency, acts as a community database. This technique is to alert people closest to the victim by using IEEE 802.15.4 standard, ZigBee wireless communication and a GPS tracking application. On triggering alert, a GSM module sends text message to individuals predefined list. Simultaneously the wireless communication module sends location details to other devices in range. While the broadcast receiver of the victims smart phone application checks for the message sent from the wearable piece on doing so the application retrieves contacts from the database for any Guardian in range.

\subsection{Existing solution in form of mobile applications}

This paper[5] presents Abhaya, an Android
Application for the Safety of Women and this app can be activated this app by a single click, whenever need arises. A single click on this app identifies the location of place through GPS and sends a message comprising this location URL to the registered contacts and also call on the first registered contact to help the one in dangerous situations. The unique feature of this application is to send the message to the registered contacts continuously for every five minutes until the "stop" button in the application is clicked. Continuous location tracking information via SMS helps to find the location of the victim quickly and can be rescued safely. This paper[6] presents SCIWARS (Spy Camera Identification And Women Attack Rescue System) intelligent alerts system which will help to prevent from being victim of any kind of attack or spy cameras. This system comprises of two parts, first part will detect Hidden cameras, which are hidden in hotels room, changing rooms. The second part will helps victims from attack like physical violence, if victims feels unsecure and helpless at any time then by pressing any key of her mobile continuously the alert message is send to nearest police control room, family, ambulance, friends which are in emergency list that alert message will contains the entire location of that victims place and images of that location which are taken by camera of her mobile.

\section{PROPOSED SYSTEM}

The proposed system comprises of a wearable device that can be embedded into a purse or clutch or also into clothes .Below figure represents the block diagram of proposed security system for personal safety.

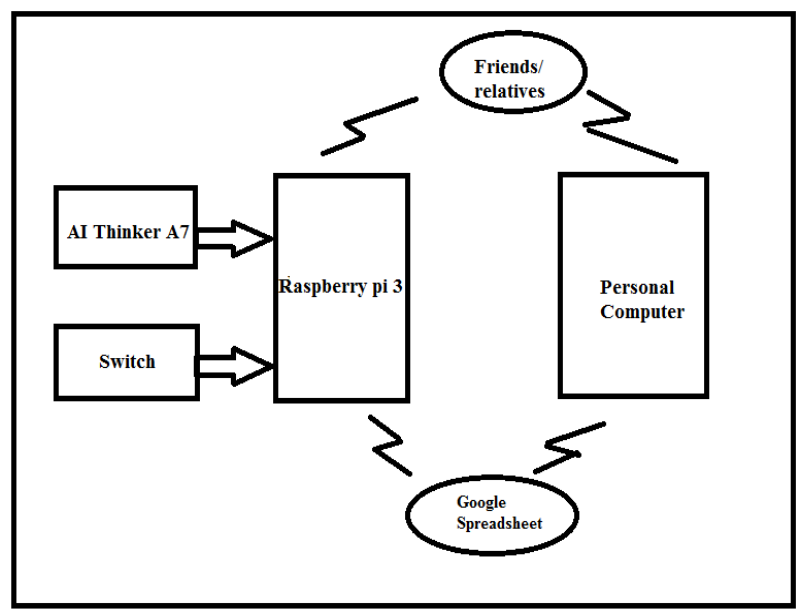

Fig 1. Block diagram of Personal Security System The device part consists of a wearable device like a bracelet. Wearable devices can be worn and forgotten about, very easy to handle and use . This wearable bracelet is integration of Rapberry Pi 3 , AI thinker A7 having GSM and GPS module on same chip and a switch for triggering the device. 
Raspberry Pi is a series of small single board computers which has evolved through various versions to improve memory capacity and peripheral device support. In this system, Raspberry Pi 3 having quad -core Cortex A53gives 10 times the performance of Raspberry pi $1,80 \%$ faster than Raspberry Pi 2 in parallelized task and provides WiFi facility is used for forwarding location of user to preset contacts and along with that, stores data to the Google cloud.

AI Thinker A7 is a GSM/GPRS/GPS function module. It supports GSM/GPRS QuadBand (850/900/1800/1900) network. Also, it supports voice calls, SMS messages, GPRS data service and GPS function. We can use it make a simple phone. The module is controlled by AT command via UART and supports $3.3 \mathrm{~V}$ and $4.2 \mathrm{~V}$ logical level.

\section{METHODOLOGY}

If device is working properly then,

- When user is in danger, will trigger the device by pressing a switch and help SMS with detail of location will be sent to predefined contact.

- Along side user's location information is getting stored continuously on google cloud.

If device not working properly / gets broken / user unable to trigger the device/internet is not available then,

- Location information was getting stored on the cloud so by use of gmail scheduler this information as an attachement will be forwarded to some predefined email Id's if the user is unable to reset the scheduled time of the email and this also ensures that user is in danger .

\section{PROTOTYPE DEVELOPED AND OUTCOMES}

Wearable device protoype is developed which can be embedded into a purse or clutch of a women or even in clothes. Fig. 2 shows the prototype developed of the device.

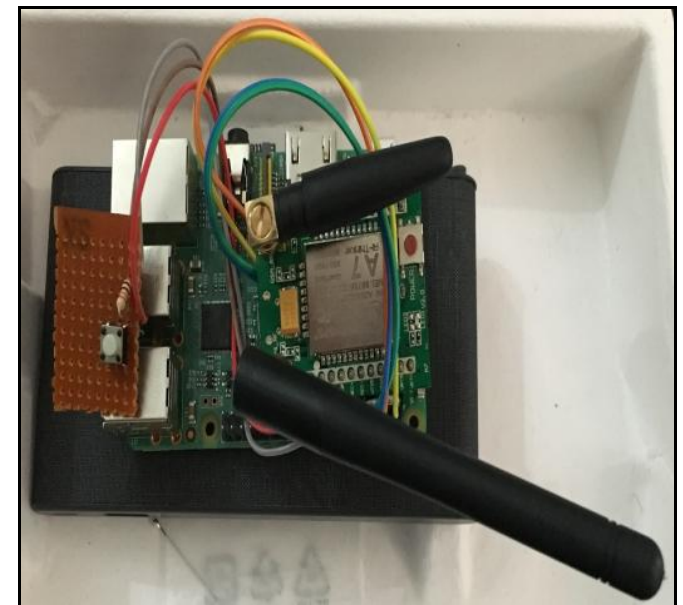

Fig 2 . Prototype of Wearable Device

Below figure shows the stored location information of the user in google spreadsheet.

\begin{tabular}{|c|c|c|c|c|c|}
\hline \multirow{4}{*}{$f_{x}$} & \multicolumn{5}{|c|}{ 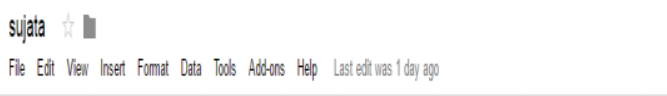 } \\
\hline & 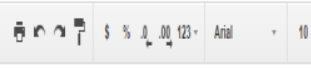 & 10. & $B I \neq \underline{A}$. & \multicolumn{2}{|c|}{ 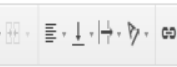 } \\
\hline & \multicolumn{5}{|l|}{ Location } \\
\hline & \multicolumn{2}{|l|}{$A$} & & & D \\
\hline$\pi$ & \multicolumn{2}{|l|}{ Location } & DateTime & |atlon & \\
\hline 72 & \multicolumn{2}{|l|}{ 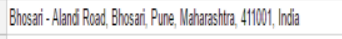 } & \multicolumn{3}{|c|}{$2017-4402131.155818 .6275 .43333,73.6804315$} \\
\hline 73 & \multicolumn{2}{|l|}{ Locedion } & DateTime & latilon & \\
\hline 74 & \multicolumn{2}{|l|}{ 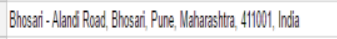 } & \multicolumn{3}{|c|}{ 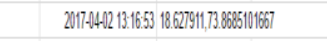 } \\
\hline 75 & \multicolumn{2}{|l|}{ Location } & DateTime & |atlon & \\
\hline 78 & \multicolumn{2}{|l|}{ 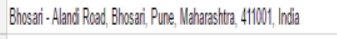 } & \multicolumn{3}{|c|}{2017.4401213 .18 .0518 .627999933337 .73 .0644282333} \\
\hline$\pi$ & \multicolumn{2}{|l|}{ Location } & DateTime & |atilon & \\
\hline 78 & \multicolumn{2}{|l|}{ 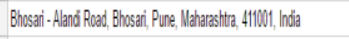 } & \multicolumn{3}{|c|}{ 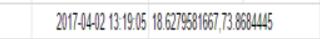 } \\
\hline 78 & \multicolumn{2}{|l|}{ Loction } & DateTime & |atlen & \\
\hline 8 & \multicolumn{2}{|l|}{ 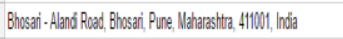 } & \multicolumn{3}{|c|}{$2017.4+212132202118.62796606667,73.6084566667$} \\
\hline 81 & \multicolumn{2}{|l|}{ Location } & DateTime & latlen & \\
\hline n & \multicolumn{2}{|l|}{ Bhosan - Alaná Road, Bhosad, Pune, Nehrarashtra, 4111001, India } & \multicolumn{3}{|c|}{ 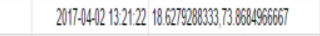 } \\
\hline s & \multicolumn{2}{|l|}{ Location } & DateTime & latlon & \\
\hline 4 & \multicolumn{2}{|l|}{ 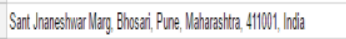 } & \multicolumn{3}{|c|}{$2017-4421213.3155318 .6221636667,73.8117733333$} \\
\hline 65 & \multicolumn{2}{|l|}{ Location } & DateTime & latlon & \\
\hline 8 & \multicolumn{2}{|l|}{ 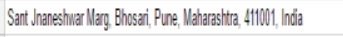 } & \multicolumn{3}{|c|}{ 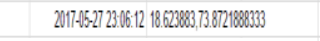 } \\
\hline
\end{tabular}

Fig 3. Stored location information of the user

When device works properly and user is able to trigger the device then an SMS is sent to preregistered numbers as shown in below figure. 


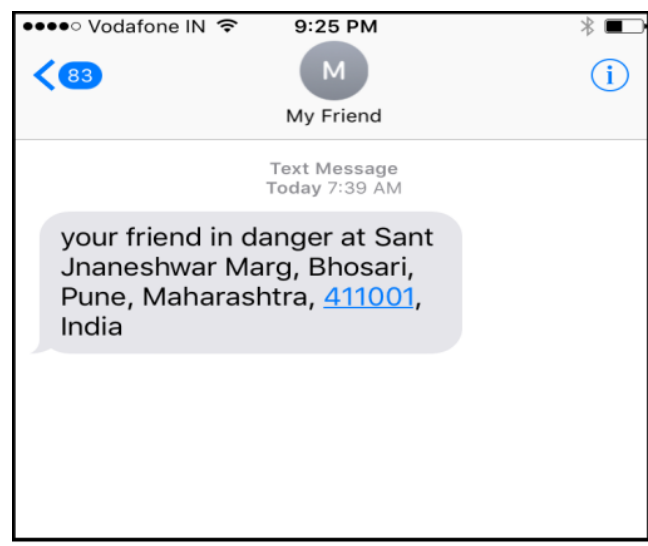

Fig.4 : SMS sent to pre-registered contacts

Below figure depicts the email scheduler screen where email is scheduled by the user .

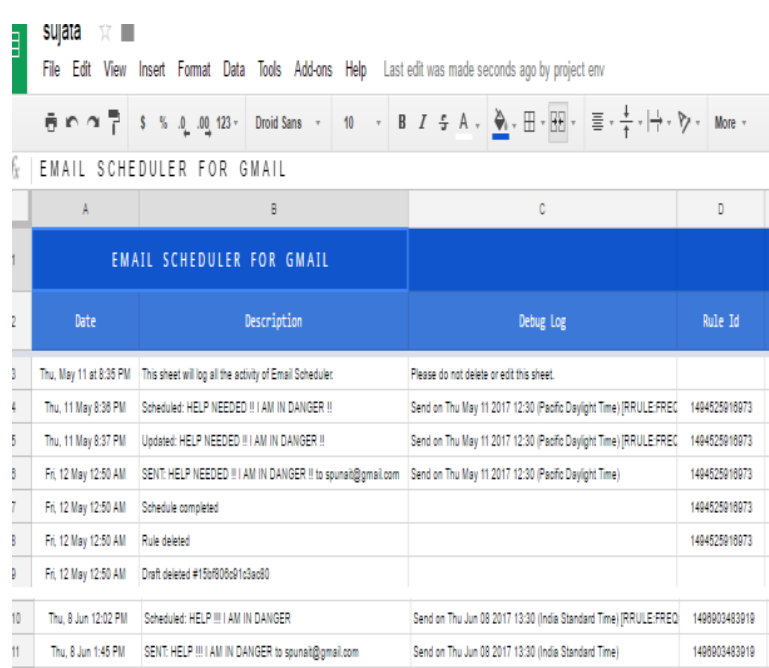

Fig 5 .Email scheduled using email scheduler

\section{ADVANTAGES}

- Easy to use as can be used as a wearable device such as it can be embedded into a purse or clutch or attached with the belt so it will not be visible and attacker may not be able to identify the device

- Ensures privacy of data as location information of person is getting stored onto google docs i.e google spreadsheet

- User relatives and friends will come to know that he/she is in danger even if he/she is unable to trigger the device or it gets broken

- Stored data on google spreadsheet will prove really useful to the police in investigation procedure as it can rely on the stored location information of the user

- This stored information speeds up the investigation procedure

\section{CONCLUSION}

Personal Security system presented in this paper will overcome the drawbacks of already available solutions in the market for safety of a child, a girl or an old age person by ensuring data is getting continuously stored to google spreadsheet and can be forwarded to the relatives or friends of user in case of danger. Solar and thermal energy harvesting techniques can be used to power the wearable device and also Raspberry pi 3 can be replaced by more appropriate component.

\section{REFERENCES}

[1]. Gowri Predeba.B et al. , "Women Security System Using GSM and GPS," International Journal of Advanced Reasearch in Engineering and Technology, Volume 3 , Issue 19, pp.247-250, April 2016

[2]. Geetha Pratyusha Miriyala et al. , "Smart Intelligent Security System for Women" International Journal of Electronics and Communication Engineering \& TechnologyVolume 7, Issue 2, pp. 41-46 ,March-April 2016

[3]. G.C. Harikiran et al. , "Smart security solution for women based on IoT," International Conference on Electrical, Electronics, and Optimization Techniques (ICEEOT), Chennai ,India - 2016

[4]. Tuman Poddar et al., "Using wearable technology to answer women's safety International Journal of Science, Technology \& Management, Volume No.04, Issue No. 05, May 2015

[5]. Ravi Sekhar Yarrabothu and Bramaramika Thota , "Abhaya :An Android App for Safety of Women," Annual IEEE India Conference (INDICON), 2015,New Delhi ,India

[6]. VaijayantiPawar et al. "SCIWARS Android App for Women Safety," International Journal of Engineering Research and Applications, Vol. 4, Issue 3( Version 1), pp.823-826,March 2014, 